Revista Brasileira de Agricultura Irrigada v.14, nº.1, p. 3816 - 3822, 2020

ISSN 1982-7679 (On-line)

Fortaleza, CE, INOVAGRI - http://www.inovagri.org.br

DOI: $10.7127 /$ rbai.v14n1001029

Protocolo 1029.20 - 12/10/2018 Aprovado em 22/05/2020

\title{
DETERMINAÇÃO DO COEFICIENTE DE CULTURA E AJUSTE DO IRRIGÂMETRO PARA O MÁNEJO DA IRRIGAÇÃO DE MILHO (ZEA MAYS L.) PARA SILAGEM
}

\author{
Eloisio de Oliveira Martins ${ }^{1}$, Ednaldo Miranda de Oliveira $^{2}$, Arthur Vargens Cardoso ${ }^{3}$
}

\begin{abstract}
RESUMO
Para qualquer cultura o manejo da irrigação tem como principal objetivo, suprir à demanda de água pelas plantas, visando à maximização da produção. Para se alcançar a máxima produtividade economicamente viável, muitas vezes, a quantidade de água a ser aplicada é menor do que a necessária para compensar totalmente a evapotranspiração da cultura $\left(\mathrm{ET}_{\mathrm{C}}\right)$, já que os custos com a irrigação se tornam reduzidos. Neste contexto, com esse trabalho objetivou-se determinar o coeficiente da cultura do milho $\left(\mathrm{K}_{\mathrm{C}}\right)$ utilizando lisímetros de drenagem e ajustar o coeficiente do Irrigâmetro (K) para o manejo da irrigação da cultura do milho. Para isso, o experimento contou com três lisímetros de drenagem. Por meio de balanço hídrico diário, determinou-se a $\mathrm{ET}_{\mathrm{C}}$ do milho. Os valores de evapotranspiração de referência $\left(\mathrm{ET}_{0}\right)$ foram obtidos com dados de uma estação meteorológica instalada no local. Também foi instalado na área experimental um Irrigâmetro que foi ajustado para determinar a evapotranspiração do Irrigâmetro $\left(\mathrm{ET}_{\mathrm{I}}\right)$. Pela relação entre a $\mathrm{ET}_{\mathrm{C}}$ e a $\mathrm{ET}_{0}$, determinou-se os valores de $\mathrm{K}_{\mathrm{c}}$ para as fases fenológicas da cultura do milho. Os valores de $\mathrm{K}_{\mathrm{c}}$ foram 1,95; 2,53; 3,42 e 4,22, para os estádios I, II, III e IV, respectivamente. Para o mesmo período analisado os valores de $\mathrm{K}_{\text {I }}$ foram 0,$94 ; 1,10 ; 0,94$ e 0,49 para os estádios I, II, III e IV, respectivamente.
\end{abstract}

Palavras-chave: Agricultura irrigada, escassez hídrica, evapotranspiração.

\section{DETERMINATION OF THE CULTURE COEFFICIENT AND ADJUSTMENT OF THE IRRIGAMETER FOR THE MANAGEMENT OF IRRIGATION OF CORN (Zea mays L.) FOR SILAGE}

\footnotetext{
ABSTRACT

${ }^{1}$ Graduado em Agronomia pelo Instituto Federal de Educação, Ciência e Tecnologia do Espírito Santo - Campus Santa Teresa (IFES-ST), Rod. ES 080, Km 93 - São João de Petrópolis, CEP 29660-000,

Santa Teresa-ES, Brasil, eloisiodeom@gmail.com

${ }^{2}$ Doutor em Recursos Hídricos e Ambientais pela Universidade Federal de Viçosa, Departamento de Engenharia Agrícola, Av. Peter Henry Rolfs, s/n - Campus Universitário, CEP 36570-900,

Viçosa - MG, Brasil, ednaldo.oliveira@ifes.edu.br

${ }^{3}$ Graduado em Agronomia pelo Instituto Federal de Educação, Ciência e Tecnologia do Espírito Santo - Campus Santa Teresa (IFES-ST), Rod. ES 080, Km 93 - São João de Petrópolis, CEP 29660-000, Santa Teresa - ES, Brasil, arthur_kardoso@hotmail.com
} 
For any culture irrigation management has as main objective, to supply the water demand for the plants, aiming at the maximization of production. To achieve maximum economically viable productivity, the amount of water to be applied is often less than that required to fully compensate crop evapotranspiration $\left(E T_{\mathrm{C}}\right)$, as irrigation costs are reduced. In this context, the objective of this work was to determine the corn crop coefficient $(\mathrm{Kc})$ using drainage lysimeters and to adjust the Irrigameter coefficient $\left(\mathrm{K}_{\mathrm{I}}\right)$ for irrigation management of corn crop. For this, the experiment had three lysimeters of drainage. By means of a daily water balance, the $\mathrm{ET}_{\mathrm{C}}$ of corn was determined. The reference evapotranspiration $\left(\mathrm{ET}_{0}\right)$ values were obtained with data from a meteorological station installed in the site. Also installed in the experimental area was an Irrigameter that was adjusted to determine the Irrigameter evapotranspiration $\left(\mathrm{ET}_{\mathrm{I}}\right)$. By the relationship between $\mathrm{ET}_{\mathrm{C}}$ and $\mathrm{ET}_{0}, \mathrm{Kc}_{\mathrm{c}}$ values were determined for the phenological phases of corn crop. Kc values were $1.95 ; 2.53 ; 3.42$ and 4.22, for stages I, II, III and IV, respectively. For the same analyzed period $\mathrm{K}_{\mathrm{I}}$ values were $0.94 ; 1.10 ; 0.94$ and 0.49 for vegetative stages I, II, III and IV, respectively.

Keywords: Irrigated agriculture, water scarcity, evapotranspiration.

\section{INTRODUÇÃO}

Devido a sua grande importância na alimentação humana, animal e fornecimento de matéria-prima para a indústria, o milho (Zea mays L.), tem sido uma das culturas de maior importância econômica estudada. Pode ser cultivado com a finalidade de produção de espigas verdes, grãos secos e silagem.

A utilização de silagem tem aumentado já que há a necessidade de se produzir alimentos volumosos para os rebanhos, visando o seu fornecimento principalmente no período seco do ano, quando as pastagens naturais se tornam mais precárias.

Considerando ainda, a baixa lâmina precipitada na região do município de Santa Teresa, ES, a produção a pasto já não é mais suficiente para garantir alimento ao rebanho, sendo a silagem de crucial importância para a mantença dos animais.

Nesse contexto, o solo se comporta como um reservatório de água, armazenando-a temporariamente e fornecendo-a as plantas à medida que necessitam. Nesse sentido é importante conhecer o efeito da deficiência hídrica em cada estádio de desenvolvimento das plantas, já que o crescimento, o desenvolvimento e a translocação de fotoassimilados encontram-se ligados à disponibilidade hídrica no solo (FANCELLI e DOURADO NETO, 2000).

Segundo Bergamaschi et al. (2004), para cultura do milho, antes de representar a aplicação de água em quantidades elevadas, a irrigação significa atender à necessidade hídrica no período crítico. Para se obter produtividades adequadas não se faz necessária a aplicação de grande quantidade de água, o que pode ser relevante em propriedades que possuem restrições quanto a mananciais ou equipamentos disponíveis.

Assim para se alcançar a máxima produtividade economicamente viável, muitas vezes, a quantidade de água a ser aplicada é menor do que a necessária para compensar totalmente a evapotranspiração da cultura $\left(\mathrm{ET}_{\mathrm{C}}\right)$, já que os custos com a irrigação se tornam reduzidos.

Visando-se determinar a $\mathrm{ET}_{\mathrm{C}}$, faz-se necessária a obtenção da evapotranspiração de referência $\left(\mathrm{ET}_{0}\right)$, a qual é determinada pelas condições ambientais do local de interesse, conjuntamente com as características fisiológicas e morfológicas da cultura, representadas por meio do seu coeficiente de cultura $\left(\mathrm{K}_{\mathrm{c}}\right)$. O $\mathrm{K}_{\mathrm{c}}$ incorpora características da planta e efeitos da evaporação do solo, variando ao longo do ciclo em função da taxa de crescimento e, consequentemente, da variação da cobertura do solo (ALLEN et al. 1998). De acordo com o mesmo autor, o coeficiente de cultura varia com o tipo de cultura, sua fase de desenvolvimento, com as condições climáticas e com os tratos culturais, sendo que, para fins práticos de manejo da água de irrigação, são considerados quatro estádios de desenvolvimento da cultura. 
A evapotranspiração pode ser obtida por medida direta ou estimada a partir de elementos climáticos, utilizando-se modelos ou métodos teóricos empíricos. O método direto está representado por diversos tipos de lisímetros, constituindo o método mais preciso, sendo o lisímetro considerado instrumento padrão para a determinação da evapotranspiração de referência (AMORIM, 1998; BERNARDO et al. 2006). Diversos lisímetros podem ser utilizados em pesquisas, sendo que neste trabalho optou-se pelo de drenagem livre para a determinação do Kc do milho.

Para se manejar a irrigação de forma adequada existem vários métodos, e o uso do Irrigâmetro é um dos mais simples. O equipamento estima a $\mathrm{ET}_{\mathrm{C}}$ e mede a quantidade de chuva precipitada, indicando, através de uma régua fabricada exclusivamente para o local em que será instalado, o momento para se iniciar as irrigações e o tempo de funcionamento do equipamento de irrigação ou a regulagem da velocidade do pivô central, sem necessidade de qualquer cálculo e em questão de segundos (SILVEIRA et al., 2009).

Desta forma, objetivou-se com este trabalho determinar o $\mathrm{K}_{\mathrm{C}}$ utilizando lisímetros de drenagem e ajustar o coeficiente do Irrigâmetro $\left(\mathrm{K}_{\mathrm{I}}\right)$ para o manejo da irrigação da cultura do milho.

\section{MATERIAL E MÉTODOS}

Este experimento foi realizado em condições de campo no Instituto Federal de Educação, Ciência e Tecnologia do Espírito Santo (IFES) - campus Santa Teresa, localizado nas coordenadas geográficas de $19^{\circ} 48^{\prime} 17^{\prime}$, latitude $\mathrm{S}, 40^{\circ} 40^{\prime} 35^{\prime}$ ' longitude $\mathrm{O}$ e altitude de $136 \mathrm{~m}$.

Ao lado da estação meteorológica, foram construídos e instalados três lisímetros com caixas de cimento amianto de 1.000L $(1,08 \mathrm{~m}$ de largura, $1,29 \mathrm{~m}$ de comprimento e $0,70 \mathrm{~m}$ de profundidade), com um dreno de PVC no fundo, preenchidos com uma camada de brita zero, sobreposta por outra de areia, seguida de solo Latossolo Vermelho-Amarelo destorroado e peneirado. Amostras do solo dos lisímetros foram submetidas à análise química e físicohídrica para determinar a densidade, textura e retenção de água em laboratório.

A fertilização do solo foi realizada de acordo com os resultados da análise química do solo e de acordo com as exigências nutricionais da cultura do milho plantado dentro dos lisímetros. O fertilizante utilizado foi o Supersimples.

Os lisímetros foram cultivados com milho no espaçamento de $0,60 \mathrm{~m}$ entre linhas e $0,25 \mathrm{~m}$ entre plantas, semeando-se linhas de mesmo espaçamento do lado externo, margeando estes lisímetros, para neutralizar o efeito de bordadura ou efeito oásis. A semeadura foi realizada manualmente. O material genético utilizado foi a varietal Capixaba Incaper 203.

As adubações nitrogenadas de cobertura realizadas de acordo com as recomendações técnicas e aplicadas manualmente de forma parcelada, distanciadas entre $5-10 \mathrm{~cm}$ da linha de semeadura, sendo $30 \%$ da dose (20 g/metro linear) aplicada 15 dias após a emergência (DAE), 30\% (20 g/metro linear) 30 (DAE), $20 \%$ (13,3 g/metro linear) aos 45 (DAE) e $20 \%$ $(13,3 \mathrm{~g} / m$ tro linear) aos 60 (DAE), correspondendo a $1105,56 \mathrm{Kg} / \mathrm{ha}^{-1}$. O fertilizante nitrogenado utilizado foi a ureia (45\% de $\mathrm{N})$.

Ao longo do desenvolvimento do trabalho foram realizados os tratos culturais necessários, seguindo recomendações técnicas exigidas para a cultura.

A evapotranspiração do milho nos lisímetros foi calculada por meio do balanço hídrico, desconsiderando a variação do armazenamento, de acordo com a expressão:

$\mathrm{ET}_{\mathrm{C}}=\mathrm{P}+\mathrm{I}-\mathrm{D}$

Em que: $\mathrm{ET}_{\mathrm{c}}$ - evapotranspiração da cultura, $\mathrm{mm} \cdot \mathrm{d}^{-1} ; \mathrm{P}$ - precipitação pluvial no lisímetro, mm; I - lâmina de água aplicada por irrigação, $\mathrm{mm}$; e D - lâmina de água drenada, mm.

As irrigações e a coleta da água percolada nos lisímetros foram realizadas com frequência de 24 horas ao longo de todo o experimento.

Os coeficientes de cultura $\left(\mathrm{K}_{\mathrm{c}}\right)$, que relacionam a evapotranspiração de determinada 
cultura com a evapotranspiração de referência em condições ótimas de umidade, fertilidade e sanidade, em seus diferentes estádios de desenvolvimento foram determinados pela equação:

$\mathrm{Kc}=\frac{\mathrm{ETc}}{\mathrm{ETo}}$

Em que: $\mathrm{ET}_{\mathrm{C}}=$ evapotranspiração da cultura, $\mathrm{mm} \mathrm{d}^{-1} ; \mathrm{ET}_{0}=$ evapotranspiração de referência, $\mathrm{mm} \mathrm{d}^{-1}$.

Os valores de $\mathrm{K}_{\mathrm{C}}$ foram determinados diariamente e agrupados no intervalo de dias de cada estádio de desenvolvimento, sendo que o valor médio para cada um representou o $\mathrm{K}_{\mathrm{C}}$ para as condições da cultura do milho para a região de Santa Teresa - ES.

A evapotranspiração de referência $\left(\mathrm{ET}_{0}\right)$ obtida com o uso do programa computacional REFET (ALLEN, 2000), aplicando-se a equação de Penman-Monteith FAO 56 (ALLEN et al., 1998) foi alimentada com os dados obtidos da estação meteorológica instalada no próprio campus.

Instalado próximo a unidade experimental e ajustado de acordo com as recomendações do fabricante para o manejo da cultura do milho, o Irrigâmetro foi utilizado para a determinação do coeficiente do Irrigâmetro $\left(\mathrm{K}_{\mathrm{I}}\right)$, que representa a razão entre a estimativa da evapotranspiração obtida no Irrigâmetro $\left(\mathrm{ET}_{\mathrm{I}}\right)$ e a evapotranspiração de referência $\left(\mathrm{ET}_{0}\right)$, determinado pela equação:

$\mathrm{K}_{\mathrm{I}}=\frac{\mathrm{ETi}}{\mathrm{ETo}}$

Em que: $\mathrm{K}_{\mathrm{I}}=$ coeficiente do Irrigâmetro, adimensional; $\quad \mathrm{ET}_{\mathrm{I}}=$ estimativa da evapotranspiração no Irrigâmetro, $\mathrm{mm} \mathrm{d}^{-1}$; e $\mathrm{ET}_{0}=$ evapotranspiração de referência, $\mathrm{mm} \mathrm{d}^{-1}$.

\section{RESULTADOS E DISCUSSÃO}

O solo dos lisímetros apresentou densidade $1,163 \mathrm{~g} / \mathrm{dm}^{3}$, analisada pelo método da proveta, textura média, avaliada conforme metodologia apresentada pela Embrapa (2011) e retenção de água realizada pelo método de campo da bacia de saturação, apresentando um teor de umidade médio na capacidade de campo de $22,64 \%$ nas duas camadas avaliadas $(0-20$ $\mathrm{cm}$ e $20-40 \mathrm{~cm}$ de profundidade).

Durante o ciclo produtivo a cultura do milho recebeu uma lâmina de água total $(\mathrm{P}+\mathrm{I})$ correspondente a 890,25 $\mathrm{mm}$, destes, $120,10 \mathrm{~mm}$ de precipitação (Figura 1), ocorrida durante o desenvolvimento do trabalho e o restante aplicado manualmente.

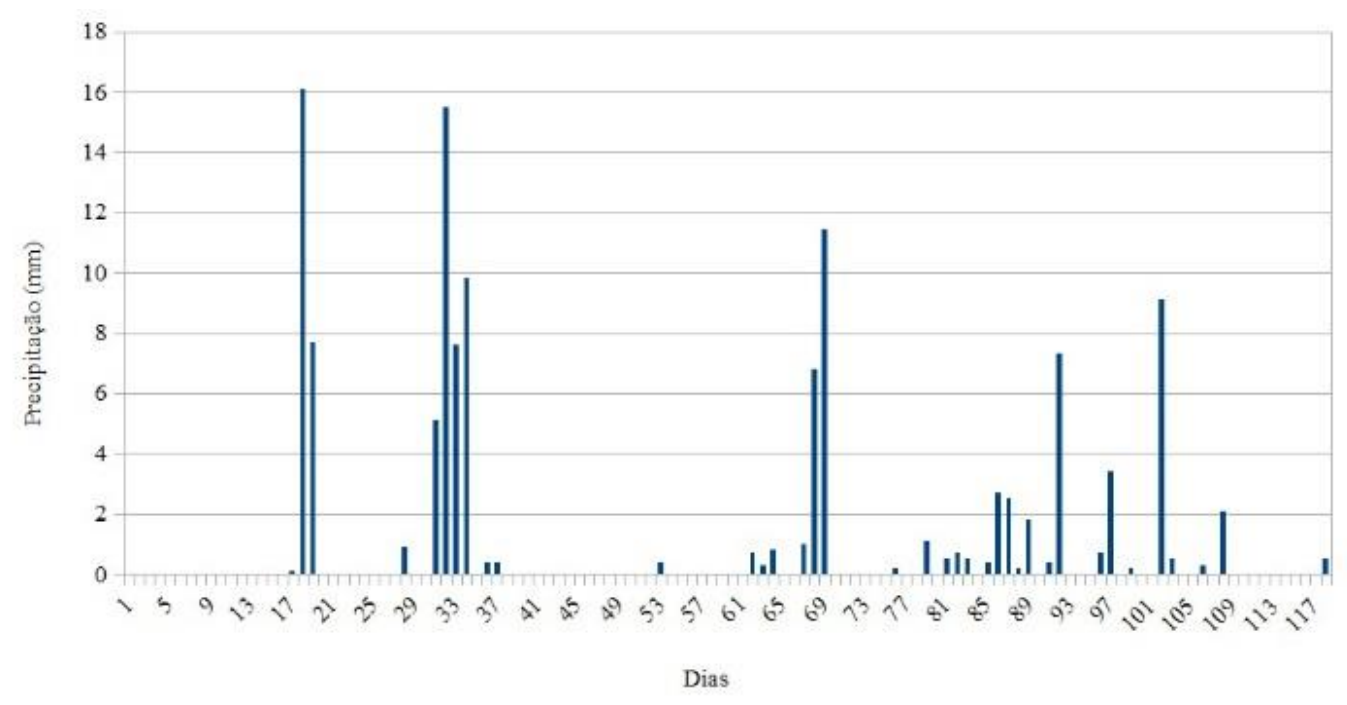

Figura 1. Precipitação (mm) ocorrida durante a execução do experimento, em Santa Teresa-ES, 2017. 
A evapotranspiração da cultura do milho $\left(\mathrm{ET}_{\mathrm{C}}\right)$ foi obtida através do balanço hídrico a partir da emergência da cultura, iniciando-se 14 dias após o plantio (DAP), e mensurada durante 104 dias após a emergência (DAE). Obteve-se durante todo o ciclo de avaliação da cultura do milho, o requerimento hídrico de $650,12 \mathrm{~mm}$, diferenciando do valor obtido por Souza et al. (2015), em trabalho sobre o requerimento hídrico e coeficiente de cultura do milho e feijão-caupi em sistema exclusivo e consorciado na região do semiárido de Pernambuco em Petrolina-PE, estes observaram para a cultura do milho em sistema consorciado um requerimento hídrico de 439,4 $\mathrm{mm}$ e de $387,1 \mathrm{~mm}$ para o sistema exclusivo e por Matzenauer et al. $\left(1998^{\mathrm{a}}\right)$ que avaliaram a evapotranspiração da cultura do milho, medida em lisímetros de drenagem em diferentes épocas de semeadura, obtiveram valores de 570 $\mathrm{mm}, 572 \mathrm{~mm}$ e $541 \mathrm{~mm}$, nas épocas de semeadura de setembro, outubro e novembro, respectivamente, nas condições climáticas do Rio Grande do Sul.

Durante todo o ciclo de cultivo, a ET $_{\mathrm{C}}$ para a c ultura do milho $(650,12$ $\mathrm{mm})$ foi superior a $\mathrm{ET}_{0}(208,47 \mathrm{~m} \mathrm{~m})$, observando durante os estádios de desenvolvimento III e IV, os maiores valores médios de requerimento híd rico para a cultura do milho durante seu ciclo de cultivo, respectivamente, 7,35 e $6,84 \mathrm{~mm}^{-1} \mathrm{~d}^{-1}$, e uma média para todo ciclo de cultivo de 6,25 mm.d ${ }^{-1}$ (Figura 2).

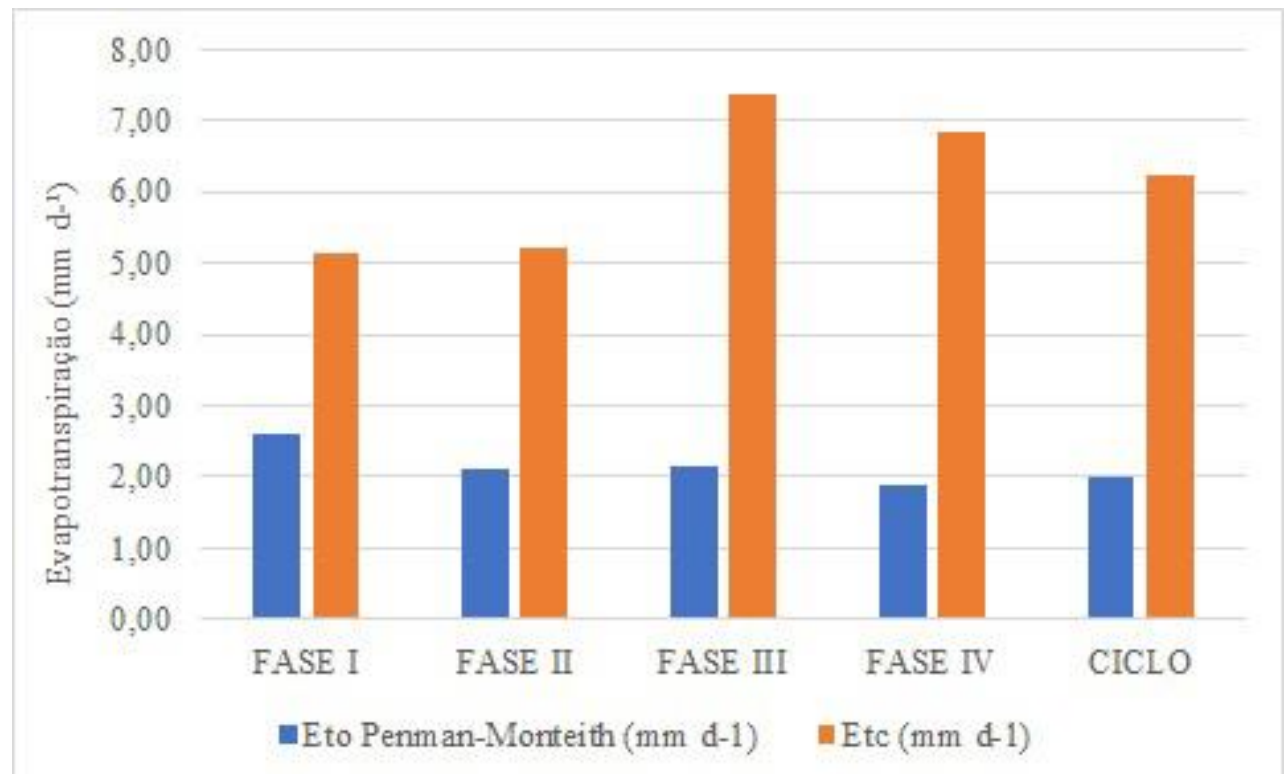

Figura 2 - Requerimento médio de água do milho (Zea mays L.), relativo a cada fase de desenvolvimento e para todo o ciclo de desenvolvimento, sob as condições climáticas do município de Santa Teresa - ES.

Utilizando a evapotranspiração de referência obtida pelo método padrão PenmanMonteith FAO 56 (ALLEN et al., 1998) calculou-se o coeficiente da cultura $\left(\mathrm{K}_{\mathrm{C}}\right)$ para a cultura do milho para a região de Santa Teresa, ES.

Na Tabela 1, observa-se que os coeficientes de cultivo obtidos pela média dos lisímetros, superestimaram aos coeficientes recomendados pela FAO para a cultura do milho em todos os estádios de desenvolvimento e ainda os encontrados por Souza et al., (2008);
Almeida, (2012) Souza et al., (2012); Santos et al., (2013).

A superestimação pode ser explicada pela variedade da cultura utilizada ou pelas características do solo como textura e estrutura e também pelas características das quais o experimento foi conduzido, ou seja, com o solo saturado, o que favorece uma maior evaporação de água do solo, assim como, uma maior evapotranspiração da cultura cultivada. 
Tabela 1. Coeficiente de cultura recomendado pela FAO 56 e determinado pela média dos lisímetros, em Santa Teresa-ES, 2017.

\begin{tabular}{ccc}
\hline Estádio & Kcb - FAO & Kc $(\mathrm{PM})$ \\
& 56 & \\
\hline I & 0,15 & 1,95 \\
II & 1,15 & 2,53 \\
III & 0,50 & 3,42 \\
IV & 0,15 & 2,39 \\
\hline
\end{tabular}

Os coeficientes do Irrigâmetro podem ser utilizados para fins práticos de manejo da água de irrigação, pois representam diretamente a conversão da evapotranspiração de referência em evapotranspiração da cultura para cada estádio de desenvolvimento, uma vez que a evapotranspiração obtida no Irrigâmetro é a própria estimativa da evapotranspiração da cultura, assim, o valor de $\mathrm{K}_{\mathrm{I}}$ se converte em $\mathrm{K}_{\mathrm{C}}$, de acordo com a equação 3 (TAGLIAFERRE et al., 2014).

A evapotranspiração da cultura do milho utilizando o coeficiente do irrigâmetro para os quatro estádios de desenvolvimento da cultura é apresentada na Tabela 2.

Tabela 2. Estimativa da evapotranspiração da cultura do milho utilizando o coeficiente do irrigâmetro (KI) para a região de Santa TeresaES.

\begin{tabular}{cc}
\hline Estádio & $\mathrm{K}_{\mathrm{I}}$ \\
\hline I & 0,94 \\
II & 1,10 \\
III & 0,94 \\
IV & 0,57 \\
\hline
\end{tabular}

Em comparação com o coeficiente da cultura $\left(\mathrm{K}_{\mathrm{c}}\right)$ recomendado pela FAO 56, o coeficiente do irrigâmetro $\left(\mathrm{K}_{\mathrm{I}}\right)$ subestimou os valores de coeficiente somente no estádio II de desenvolvimento, estádio este que coincidiu com os maiores índices pluviométricos 34,1 mm e com a menor taxa de radiação solar média diária de 10886,6 Kj/m² d, superestimando nos demais estádio de desenvolvimento da cultura do milho I, III e IV, o coeficiente da cultura recomendado pela FAO 56 (Tabela 1). O nível de água no evaporatório do irrigâmetro utilizado para a determinação do coeficiente do irrigâmetro dos estádios de desenvolvimento da cultura foi 2,5; 3,5; 4,5 e 3,0 para os estádios de desenvolvimento I, II, III e IV, respectivamente.

\section{CONCLUSÃO}

Os valores de $\mathrm{K}_{\mathrm{c}}$ utilizando lisímetros de drenagem para os estádios de desenvolvimento da cultura do milho I, II, III e IV, foram respectivamente 1,$95 ; 2,53 ; 3,42$ e 2,39.

A evapotranspiração da cultura do milho utilizando o coeficiente do irrigâmetro $\left(\mathrm{K}_{\mathrm{I}}\right)$ para os estádios de desenvolvimento da cultura do milho I, II, III e IV, foram respectivamente 0,$94 ; 1,10 ; 0,94$ e 0,57 .

\section{AGRADECIMENTOS}

Ao Instituto Federal de Educação, Ciência e Tecnologia do Espírito Santo Campus Santa Teresa pelo apoio à pesquisa e com a infraestrutura oferecida para a condução do experimento.

Ao Instituto Federal de Educação, Ciência e Tecnologia do Espírito Santo no desenvolvimento deste trabalho, através da concessão de bolsa de pesquisa.

\section{REFERÊNCIAS BIBLIOGRÁFICAS}

\section{ALLEN, R. G. REF-ET: Reference evapotranspiration calculator, Version 2.1. Idaho: Idaho University, 2000. 82p.}

ALLEN, R.G. et al. Crop evapotranspiration - Guidelines for computing crop water requirements. FAO. Irrigation and Drainage Paper, No. 56, FAO, Rome. 1998.

ALMEIDA, B. M. Evapotranspiração, coeficiente de cultura e produção do milho sob condições de salinidade residual. Mossoró, RN. 2012.

AMORIM, M. C. de. Avaliação da eficácia do lisímetro de lençol freático constante, do tanque classe "A" e do modelo de PenmanMonteith (FAO) para estimativa da 
evapotranspiração de referência (ET0). Dissertação Mestrado em Meteorologia Agrícola. 1998. Universidade Federal de Viçosa, Viçosa. Minas Gerais, Brasil. 56 p

BERGAMASCHI, $H$. et al. Distribuição hídrica no período crítico do milho e produção de grão. Pesquisa Agropecuária Brasileira, Brasília, v.39, n.9, p.831-839, 2004.

BERNARDO, S.; MANTOVANI, E. C. e SOARES, A. A. 2006. Manual de irrigação. Editora Universidade Federal de Viçosa. 7a. ed. Viçosa, Minas Gerais, Brasil. 611p.

EMBRAPA. Manual de métodos de análise de solos. Rio de Janeiro: Embrapa Solos, 2011. $2^{\mathrm{a}}$ ed. $95 \mathrm{p}$.

FANCELLI, A.L.; DOURADO NETO, D. Ecofisiologia e fenologia. In: Produção de milho. Guaíba: Agropecuária, 2000. p.21-53.

MATZENAUER, R.; BERGAMASCHI, $\mathrm{H}$; BERLATO, M. A.; MALUF, J. R. T. Evapotranspiração da cultura do milho. I Efeito de épocas de semeadura. Revista Brasileira de Agrometeorologia, Santa Maria, v. 6, n. 1, p. 9 - 14, 1998.

SANTOS, W. O; NUNES, R. L. C.; GALVÃO, D. C.; PEREIRA, V. C.; MANIÇOBA, R. M.; LIMA, J. G. A.; VIANA, P. C. Evapotranspiração da cultura do milho verde, análise estatística. Agropecuária Científica no
Semi-Árido, V. 9, n. 1, p. 75 - 81, jan - mar, 2013.

SILVEIRA, P. M.; RAMOS, M. M.; OLIVEIRA, R. A. 2009. CircularTécnica, 84. Manejo da Irrigação do Feijoeiro com o Uso do Irrigâmetro. Santo Antônio de Goiás. 1 ${ }^{\circ}$ Edição. ISSN 1678-9636.

SOUZA, A. P.; DE LIMA, M. E.; DE CARVALHO, D. F. Evapotranspiração e coeficientes de cultura do milho em monocultura e em consórcio com a mucunacinza, usando lisímetros de pesagem. Revista Brasileira de Ciências Agrárias. v. 7, n.1, p. 142-149, jan - mar., 2012.

SOUZA, L. S. B.; MOURA, M. S. B.; SEDIYAMA, G. C.; DA SILVA, T. G. F. Requerimento hídrico e coeficiente de cultura do milho e feijão-caupi em sistemas exclusivo e consorciado. Revista Caatinga, Mossoró, v. 28, n 4, out-dez., 2015. p. 151 - 160

SOUZA, L. S. B.; MOURA, M. S. B.; SEDIYAMA, G. C.; SILVA, T. G. F.; BRANDÃO, E. O. Determinação do coeficiente de cultura do milho (Zea mays $L$.) sob condições de semiárido brasileiro. 2008.

TAGLIAFERRE, C.; SANTOS, L. D C.; NETO, I. J. D. S.; SANTOS, T. J.; ROCHA, F. A.; SILVA, C. S. Ajuste e aplicação do irrigâmetro no manejo da água de irrigação. Irriga, Botucatu, v. 19, n. 1, janeiro-março, 2014. p. 61-72 